\title{
A probiotic fermented dairy product improves immune response to influenza vaccination in the elderly
}

\author{
J. Aubin ${ }^{1}$, M. Remigy ${ }^{2}$, L. Verseil ${ }^{3}$, R. Bourdet-Sicard ${ }^{3}$, S. Vaudaine $^{3}$, S. van der Werf ${ }^{1}$ \\ and S. I. Samson ${ }^{3}$ \\ ${ }^{1}$ Pasteur Institute, Paris, France, ${ }^{2}$ Maison de Retraite Sainte Famille, Metz, France and ${ }^{3}$ Danone Research, \\ Palaiseau, France
}

The elderly respond poorly to influenza vaccination compared with younger adults ${ }^{(1)}$. The aim of the study was to investigate whether daily consumption of a probiotic dairy product (Actimel ${ }^{\mathbb{R}}$; Groupe Danone, Paris, France) could improve vaccination responses in the elderly.

In a multicentre placebo-controlled randomized double-blind study eighty-six elderly subjects (mean age 83 years) received Actimel ${ }^{\circledR}$ or acidified milk for 7 weeks. Consumption of the dairy product started 4 weeks before vaccination. The specific antibody levels against the three viral strains $(\mathrm{H} 1 \mathrm{~N} 1, \mathrm{H} 3 \mathrm{~N} 2$ and $\mathrm{B})$ comprising the vaccine were measured 3 weeks after vaccination by a haemagglutination inhibition test, a semi-functional methodology validated by the WHO for influenza antibody measurement ${ }^{(2)}$.

Antibody levels against the three influenza strains were increased in elderly subjects consuming the probiotics compared with those consuming the acidified milk; however, these differences were not significant. A trend in favour of the probiotic group was also observed in relation to seroprotection against the H1N1 strain (64\% of the subjects consuming probiotics showed seroprotection compared with $42.5 \%$ in the control group; $P=0.08$ ). Interestingly, two subpopulations were more responsive to the probiotic effect during vaccination, the free-living elderly and elderly women. In the free-living elderly population the probiotic dairy product significantly improved seroprotection against the $\mathrm{H} 3 \mathrm{~N} 2$ strain $(16.2 \%$ more subjects in the probiotic group were $\mathrm{H} 3 \mathrm{~N} 2$-seroprotected compared with the control group; $P=0.02)$ and significantly improved $\mathrm{H} 3 \mathrm{~N} 2$ seroconversion $(27.3 \%$ more subjects in the probiotic group were H3N2-seroconverted compared with the control group; $P=0.05$ ). Among the women the probiotic group showed a significant increase in seroprotection against H1N1 (41.9\% more subjects in the probiotic group were seroprotected; $P=0.02)$ and a significant increase in H1N1 seroconversion $(28 \%$ more subjects in the probiotic group were seroconverted; $P=0.02$ ).

The present study suggests that daily consumption of Actimel ${ }^{\circledR}$, a probiotic fermented dairy product, can improve immune responses to influenza vaccination, and in particular seroprotection in a population reacting poorly to the vaccination.

1. Cox RJ, Brokstad KA \& Ogra P (2004) Scand J Immunol 59, 1-15.

2. World Health Organization (2005) Weekly Epidemiological Record no. 33, p. 283. Geneva: WHO; available at http://www.who.int/wer/2005/ wer8033.pdf 